\title{
Preterm prelabor rupture of membranes and severe oligohydramnios from 22-34 weeks of Amenorrhea
}

\author{
Patient Initials: N.M Age: 36years \\ IP Number: 0018450 Address: Zana \\ N.O.K: S.D (Husband) Tribe: Muganda \\ Religion: Catholic Occupation: Retail business \\ DOA: 18/9/2014 DOD: 27/9/2014
}

A G5P4+0, LNMP 24/01/2014, EDD 31/10/2014, WOA 33W6D; 33 weeks by scan done on 30th June 2014 ( 22 weeks then)

\section{Presenting complaint}

Clear watery vaginal discharge for 3 months.

Lower abdominal pain for 5 days.

\section{History of presenting complaint}

She had earlier been admitted to Nsambya hospital on 30th June 2014 with the complaint of vaginal discharge for three days without other symptoms. At that time, she reported that she was asleep when she suddenly felt her bed wet. The fluid was coming from the vagina, it was clear, not blood stained, not mucoid and odorless. It was not associated with abdominal pains, fever or other genito- urinary symptoms. There was no history of trauma.

The ultrasound scan done on 30th June 2014 indicated a single live fetus at 22 weeks with severe oligohydramnios. The AFI was $3 \mathrm{~cm}$, Urinalysis was normal. She stayed in the hospital under conservative management of bed rest, pad checks and 48hourly Complete blood counts. She was later discharged at 23.6Weeks of amenorrhea on erythromycin $500 \mathrm{mg}$ every $6 \mathrm{hrs}$ for 5 days and was scheduled to attend the High risk antenatal clinic. She was counselled on the danger signs and what to lookout for. She continued to have on and off mild drainage of liquor without any other symptoms for the next two months. She had three antenatal visits at hospital after discharge and serial ultra sound scans monthly which showed reduced liquor. She was given one dose of malaria prophylaxis, and hematinics. She did not continue with any oral antibiotics after stopping the erythromycin. She also abstained from sexual intercourse during the entire period.

Three days prior to the re-admission, she developed progressive dull lower abdominal pain with a vaginal discharge but no bleeding. She had normal micturition habits, no fever, and no history of trauma. This is what prompted her to come to hospital otherwise she had planned to return when she is at term.

\section{Review of other systems did not reveal signi- ficant findings \\ Past obstetric history}

The previous four pregnancies were all carried to term with no major antenatal concerns. The first was delivered by spontaneous vaginal delivery at Mulago hospital in 2001 and is alive. The second was delivered by assisted breech delivery in 2005 . The neonate was

\author{
Volume 2 Issue 5 - 2017
}

\author{
Eleanor Nakintue,' Daniel Murokora, ${ }^{2}$ Daniel \\ Zaake, ${ }^{3}$ Peter Sekweyama ${ }^{4}$ \\ 'St. Francis Hospital, Uganda \\ ¿Uganda Women's Health Initiative, Uganda \\ ${ }^{3}$ Academic coordinator, Mother Kevin Post graduate school- \\ Nsambya hospital, Uganda \\ ${ }^{4}$ Dean of medical school, Uganda Martyrs University Nkozi, \\ Uganda
}

\begin{abstract}
Correspondence: Daniel Murokora, Medical director, Consultant Gynaecologist/Obstetrician, Uganda Women's Health Initiative, P.O Box 32275, Kampala, Uganda,

Email murokora@gmail.com
\end{abstract}

Received: May II, 2017| Published: August 23, 2017

asphyxiated and died after one hour. The third was delivered by EmCS due to fetal distress in 2009. The neonate died after 48hours. The fourth pregnancy was also delivered by EmCS in Nsambya hospital due to failed trial of labor in 2012 . The birth weight was $3.0 \mathrm{~kg}$ with good outcome.

\section{Past gynecological history}

There was a history of sexually transmitted infection which presented as purulent offensive vaginal discharge before she conceived the current pregnancy and was treated with unknown medication. There was no history of any gynecological procedures done. She has never been screened for cervical cancer.

\section{Menstrual history}

The menarche was at $15 y$ ears. The cycle was 26 days and she bled on average four days. She reported mild dysmenorrheal.

\section{Contraceptive history}

She used both natural methods and Depo-Provera to space the first two children. There after she used implants.

\section{Past medical history}

She reported being allergic to metronidazole. There were no other significant findings.

\section{Past surgical history}

Except for two EmCS, there were no other surgical procedures. There was no known history of blood transfusion.

\section{Family history}

There was no reported history of pre-term rupture of membranes, preterm delivery or connective tissue diseases such as rheumatoid arthritis. 


\section{Social history}

She is married in a monogamous relationship. Her level of education is secondary. She runs a small retail business. She does not smoke or drink alcohol. The husband smokes tobacco cigarettes. She does not use recreational drugs.

Summary: NM 36years old G5P4+0 with two previous scars at 33 WOA and prolonged rupture of membranes, for 8 weeks.

\section{Examination findings}

\section{General exam}

She was in a good general condition. She had no pallor of mucous membranes, no jaundice, or edema.

Axillary temperature was $36.00 \mathrm{C}$

\section{Cardiovascular exam}

The pulse rate was $68 \mathrm{bpm}$; Blood pressure $100 / 60 \mathrm{mmHg}$.

The JVP was normal and the heart sounds were normal.

Respiratory system: She was breathing calmly at rest. The respiratory rate was 20 breaths/min and the lung fields had normal air entry without added sounds.

Per abdomen: The abdomen was distended with a mid-subumbilical scar. The fundal height corresponded to 36weeks. The presentation was cephalic with longitudinal lie. The descent was $5 / 5$ abdominally. The scar was non-tender and no contractions were palpable in 10 minutes. The fetal heart was $144 \mathrm{bpm}$ and regular.

Speculum vaginal exam: The vulva and vagina were normal.

The cervix was long, central and closed. There was a slight nonfoul watery discharge from the cervical os upon coughing.

\section{Impression}

G5P4+0 with 2 previous scars and 2 live babies with prolonged preterm prelabor rupture of membranes

Plan of management.

The patient was admitted to the antenatal ward.

Intra Muscular dexamethasone $6 \mathrm{mg} 12$ hourly for 48 hours was administered

The following investigations were requested:

a. Obstetric scan (for Amniotic fluid index, biophysical score and placental location);

b. Complete blood count.

c. Blood grouping and cross-matching.

The obstetric scan results indicated a live fetus in cephalic presentation at 36 weeks. There was no measurable liquor and the placenta was fundo-posterior. The biophysical score was $6 / 8$ with 0 for liquor volume. The $\mathrm{CBC}$ picture showed a leucocyte count of 10,000/ml, neutrophils 75\%, Hb 10.8g/dl, and Platelets 138,000/ml.

The blood group was AB RhD positive.

Based on the obstetric scan results, an EmCS was scheduled after cross-matching two units of whole blood. Intravenous ceftriaxone, $2 \mathrm{~g}$ stat was given as prophylactic antibiotics.

Under spinal anesthesia, the abdomen was accessed through mid-sub umbilical incision by removing the old scar tissue. A lower transverse incision made on the uterus to deliver a live baby boy with an Apgar score of 8-10, birth weight 2.6kg with bilateral club foot and fused cranial sutures. The placenta was fundal and an attempt at delivery by controlled cord traction was made but only half of it was delivered. The remaining piece was adherent to the fundus so a diagnosis of placenta accreta was immediately made.

The specialist on duty was immediately consulted while the uterine vessels were clumped and the fundus was packed. The decision to do a supra cervical caesarian hysterectomy was made and a sub-total hysterectomy was done with preservation of both ovaries and tubes. The surgery was uneventful with a blood loss estimate of $1000 \mathrm{ml}$ and discharge vitals to High Dependence Unit (HDU) were BP $100 / 58 \mathrm{mmHg}$, the pulse rate of $106 \mathrm{bpm}$ and the patient was awake with $\mathrm{SPO} 2$ of $95 \%$ on room air. The urine output was $500 \mathrm{ml}$-clear urine.

Routine post-operative care was instituted in HDU with no major events observed. She was discharged to postnatal ward after 12 hours to continue IV ceftriaxone 2g OD; IV metronidazole 500mg 8hrly for 3 days; IM tramadol $100 \mathrm{mg} 8 \mathrm{hrly}$ for 3 doses. The discharge status was mild anemia, BP $100 / 65 \mathrm{mmHg}$ with a pulse rate of $98 \mathrm{bpm}$ and SPO2 of $97 \%$ on room air.

While on postnatal ward, the patient remained stable, afebrile with normal abdominal examination. The bowel sounds were present at 24 hours' post-operation. The urethral catheter was removed after 24hours after satisfactory urine output of $2000 \mathrm{ml}$ in 24hours. A soft diet was commenced and she opened bowels after 36hours. A complete blood count was done on the second post-op day to check the hemoglobin level. The $\mathrm{Hb}$ was $8.5 \mathrm{~g} / \mathrm{dl}$ with a leucocyte count of $11,000 / \mathrm{ml}$ and a neutrophil count of $78 \%$.

The second, third and fourth days were also uneventful and a post-discharge plan was made after discussing with her the operative findings. The neonate showed no signs of respiratory distress and was breast feeding normally for the entire period under observation. An orthopedic referral was made to review the newborn.

She was discharged after 5 days in a stable state.

Discharge recommendations:

a. Fefol $200 \mathrm{mg} / 5 \mathrm{mg}$ once daily for one month.

b. Follow up appointment after 6 days.

c. Cervical cancer screening periodically.

At review she had mild abdominal pain since discharge but no fever or PV discharge. She had mild pallor. The abdomen was soft, non-tender with a healed incision. The baby was stable in orthopedic boots. The breathing was okay. She was allowed home to continue hematinics and Paracetamol was added for 3days for pain. She was to be reviewed after 4weeks but she did not return.

\section{Case discussion}

\section{Introduction}

Preterm prelabor rupture of membranes (PPROM) is the rupture 
of membranes before 37 WOA.PPROM occurs in 3percent of pregnancies and is responsible for, or associated with, approximately one-third of preterm births. PROM before 27weeks' gestation occurs in approximately $0.5 \%$ of all pregnancies. ${ }^{1}$ It is associated with severe complications, such as premature birth, pulmonary hypoplasia and fetal death. ${ }^{2}$ Placenta accreta as a risk factor for PPROM has only been documented in a few case reports ${ }^{3}$ Prolonged ROM is any ROM that persists for more than 24 hours and prior to the onset of labor as what happened in this patient.

\section{The risk factors include previous PPROM, genital in- fection, cigarette smocking}

Making a diagnosis: Maternal history and physical examination are the most crucial. From my clinical practice, usually the woman will report a feeling or sensation of sudden gush of fluid from the vagina to her thighs with no urge to pass urine and having no control over the fluid. Often times, these women report waking up in bed and finding their beddings wet. A small percentage, especially the prime gravidas usually may not realize they are draining when the leak is on and off and they may think it is increased vaginal discharge of pregnancy.

Upon arrival in hospital, and history taking, the vagina and vulva may obviously be wet with fluid in some cases. If this isn't the case, we insert a sterile speculum to visualize the cervix and may request the woman to cough and provoke a drain from the cervix.

A small amount of vaginal fluid is then got and put on a slide after mixing with potassium hydroxide. This mixture is visualized under a microscope for a ferning appearance which will confirm that the fluid is intrauterine. This can be routinely done on the wards if we have a microscope.

If PROM is not obvious after visual inspection, the diagnosis can be confirmed by testing the $\mathrm{pH}$ of the vaginal fluid, which is easily accomplished with nitrazine paper. Amniotic fluid usually has a $\mathrm{pH}$ range of 7.0 to 7.3 compared to the normally acidic vaginal $\mathrm{pH}$ of 3.8 to 4.2. False-negative and false-positive nitrazine test results occur in up to 5 percent of cases. False negative tests results can occur when leaking is intermittent or the amniotic fluid is diluted by other vaginal fluids. False positive results can be due to the presence of alkaline fluids in the vagina, such as blood, seminal fluid, or soap. In addition, the $\mathrm{pH}$ of urine can be elevated to near 8.0 if infected with Proteus species.

In developed settings an absorbent pad (AmnioSense) that changes color at $\mathrm{pH}>5.2$ is used as a panty liner and marketed to pregnant women. This is done when the woman is not going to be admitted in hospital. Instillation of indigo carmine in equivocal cases, instillation of indigo carmine into the amniotic cavity can be considered and usually leads to a definitive diagnosis. Under ultrasound guidance, $1 \mathrm{~mL}$ of indigo carmine in $9 \mathrm{~mL}$ of sterile saline is injected transabdominally into the amniotic fluid and a tampon is placed in the vagina. One-half hour later, the tampon is removed and examined for blue staining, which indicates leakage of amniotic fluid. This test is rarely indicated, given the availability of the non-invasive AmniSure test and the risks of amniocentesis (eg, rupture of membranes, fetal injury).

Placental alpha microglobulin-1 protein assay (AmniSure). This is a rapid slide test that uses immunochromatographic methods to detect trace amounts of placental alpha microglobulin-1 protein in vaginal fluid. An advantage of this test is that it is not affected by semen or trace amounts of blood.
The management of PPROM is among the most controversial issues in perinatal medicine. Because of the following points that will be discussed briefly in relation to this patient.

i. Expectant management versus intervention.

ii. Use of tocolytics.

iii. Duration of administration of antibiotic prophylaxis.

iv. Timing of administration of antenatal corticosteroids.

v. Methods of testing for maternal/fetal infection.

vi. Timing of delivery.

\section{Previable PPROM and pregnancy outcome}

Previable PPROM is defined as the spontaneous drainage of liquor before 24weeks of gestation. It is Previable because there is no significant alveolar development to facilitate extra-uterine fetal survival.

In one retrospective study of 35 patients with previable rupture of membranes below 24weeks, the latency period ranged from 0 to 137 days, with an average of 35 days. The amniotic fluid was reduced in $75 \%$ of the cases. Expectant management was adopted in $86 \%$ of the cases. Clinical chorioamnionitis occurred in $71 \%$, being three times more frequent in parous women. Bacteriuria was present in $6.6 \%$. Two women developed laboratorial and clinical signs of sepsis, none of them needed hysterectomy. There were no maternal deaths. Mean gestational age at delivery was 24 weeks, ranging from 16 to 39 weeks. In the expectant group, preterm delivery rate was $68 \%$. There was one case of abruption. Cesarean rate was $31 \%$. Neonatal mortality was $42 \%$. Overall neonatal survival was $35 \% .{ }^{4}$

In another retrospective cohort of 336 patients with PPROM below 27 weeks $21 \%$ neonates were born within 48 hours after PPROM, whereas $17 \%$ neonates were still unborn 50days after PPROM. The median interval between PPROM and delivery was 10days. Of all neonates, $71 \%$ were born before 27 weeks and only $2.4 \%$ after 37 weeks. The overall perinatal mortality rate was $49 \%$, of which $28 \%$ were stillbirths. Of the 170 surviving neonates (alive at seven days after birth), $41 \%$ suffered serious morbidity (RDS grade 3 or 4 , IVH, NEC, CLD or (suspicion of) sepsis). Some neonates had multiple morbidities. $59 \%$ of the surviving neonates survived without any of these endpoints, which is $30 \%$ of the total group of 336 neonates. ${ }^{5}$

According to other studies on preterm PROM, clinically evident intra-amniotic infection occurs in $13-60 \%$, and postpartum infection occurs in $2-13 \% .^{6}$ The incidence of infection increases with decreasing gestational age at membrane rupture. ${ }^{7}$ Digital vaginal exam should not be done in cases of PROM because this equally increases the incidence of intrauterine infections. ${ }^{8}$ Fetal mal-presentation is increased with preterm PROM. Abruptio placenta affects 4-12\% of pregnancies with preterm PROM. ${ }^{9}$ However, serious maternal sequelae are uncommon. ${ }^{10}$

The effect of latency on perinatal outcome is not entirely clear and the effects of a prolonged latency period do not seem to be consistent across gestational ages. ${ }^{11}$ In the study by Jantien L et al. ${ }^{4}$ latency did not lead to an improvement in perinatal survival. This may be explained by the fact that although the latency period in the group with the earliest PPROM appeared to be the longest, many of these fetuses could not benefit from this latency as $70 \%$ were born 
before 27 weeks and $59 \%$ even before 24 weeks. The earlier the GA at PPROM, the higher the perinatal mortality rate $(71 \%$ in subgroup PPROM 13-20weeks versus $27 \%$ in subgroup 24-27weeks).

At all gestational ages before term, respiratory distress has been reported to be the most common complication of preterm birth. ${ }^{12}$ Other serious forms of morbidity, including neonatal infections, intraventricular hemorrhage, and necrotizing enterocolitis, are also common remote from term. Preterm PROM and exposure to intrauterine inflammation have been associated with an increased risk of neuro developmental impairment. ${ }^{13}$ Early gestational age at membrane rupture also has been associated with an increased risk of neonatal white matter damage $(\mathrm{P}<.001)$, after controlling for corticosteroid administration, latency interval, gestational age at delivery, and birth weight. ${ }^{14}$

However, no data exist that suggest immediate delivery after presentation with PROM will avert these risks.

Reported risks of pulmonary hypoplasia after PROM at 16-26 weeks of gestation vary from less than $1 \%$ to $27 \% .{ }^{15}$ Lethal pulmonary hypoplasia rarely occurs with membrane rupture subsequent to 24 weeks of gestation, presumably because alveolar growth adequate to support postnatal development already has occurred. ${ }^{16}$ Early second trimester membrane rupture, severe oligohydramnios, and duration of membrane rupture longer than 14days are primary determinants of the risk of pulmonary hypoplasia. ${ }^{17}$ Prolonged oligohydramnios also is associated with in utero deformation, including abnormal facies (i.e., low-set ears and epicanthal folds) and limb contractures and other positioning abnormalities.

Overall, the prognosis for perinatal survival and morbidity after early PPROM seems poor, with survival rates between $14 \%$ and $70 \%{ }^{18}$ Outcomes of survivors of preterm PROM depend on the gestational age, presence of infection, length of latency, and other maternal and fetal complications. Infection, cord accident, and other factors contribute to the $1-2 \%$ risk of antenatal fetal demise after preterm PROM

Significant maternal complications after second trimester and previable PPROM have been reported to include intra-amniotic infection, endometritis, abruptio placentae, retained placenta, and postpartum hemorrhage. Maternal sepsis is a rare but serious complication reported in approximately $1 \%$ of cases, and isolated maternal deaths due to infection have been reported in this setting.

\section{Management of pre-viable PPROM}

According to ACOG 2007 practice guidelines, the patient who experiences PROM between 24weeks and 31completed weeks of gestation should be cared for expectantly if no maternal or fetal contraindications exist until 33completed weeks of gestation. Prophylaxis use of antibiotics to prolong latency and a single course of antenatal corticosteroids can help reduce the risks of infection and gestational age-dependent neonatal morbidity.

At less than 24 weeks of gestation the following is recommended:

a) Patient counseling on prognosis.

b) Expectant management or induction of labor dependent on patient informed choices.

c) Group B streptococcal prophylaxis is not recommended. d) Corticosteroids are not recommended.

e) Antibiotics, there are incomplete data on use in prolonging latency.

In determining whether to continue the pregnancy or terminate, the combination of birth weight, gestational age, and sex provide the best estimate of chances of survival and should be considered in individual cases.

\section{Should patients with pre-viable PPROM be managed at home?}

Although no evidence or consensus of opinion exists regarding the benefit of an initial period of inpatient observation in these patients, this approach may include strict bed and pelvic rest to enhance the opportunity for resealing, as well as early identification of infection and abruptio placenta if expectant management is pursued. In addition to clinical follow-up, it may be useful to instruct patients to abstain from intercourse, limit their activities, and monitor their temperatures. Prematurity is the principal risk to the fetus, while infection morbidity and its complications are the primary maternal risks. All plans for management of PPROM remote from term should include the family and the medical team caring for the pregnancy, including the neonatal and maternal medical team. Remote from term, PPROM should only be cared for in facilities where a Neonatal Intensive Care Unit (NICU) is available and capable of caring for the neonate. Because most PPROM pregnancies deliver within a week of ROM, transfer of the pregnant mother to a qualified facility is urgent and should be facilitated immediately upon diagnoses.

Typically, women with pre-viable PROM who have been cared for as outpatients are readmitted to the hospital for bed rest and observation for infection, abruption placentae, labor, and nonreassuring fetal heart rate patterns once the pregnancy has reached the limit of viability (28weeks for low resourced settings where neonatal care is still inadequate). Administration of antenatal corticosteroids for fetal maturation is appropriate at this time given that early delivery remains likely.

\section{This patient in perspective}

This patient had pre-viable PPROM and severe oligohydramnios from 22-34weeks which were critical markers against good fetal outcome. In Nsambya hospital, women with such a presentation are admitted on the wards and counselled for prognosis. Performing fetal lung maturity tests is expensive in our setting but would guide management to improve neonatal survival and reduce on the risk of skeletal deformities that result from prolonged oligohydramnios which occurred in this neonate.

The cause of pre-viable PPROM was not detected although it would have been prudent to do vaginal cultures for GBS at that time. Accreta as the cause of the PPROM can only be speculative as there were no other causes identified. Although the risk of intrauterine infection is high in prolonged PPROM, she did not experience it. This is difficult to explain, but perhaps abstinence from coitus and the low gestational age at PPROM contributed positively.

The risks and potential benefits of expectant management were discussed with the patient and her family, and informed consent was obtained. The maternal and fetal status were reevaluated daily, and the safety and potential benefits of expectant management discussed. 
Spontaneous sealing of the membranes does occur occasionally $(<10 \%$ of all cases), but this did not occur in this patient. Amniocentesis can provide information about lung maturity accuracy and correctness of the diagnoses of PROM and infection. In developed settings membrane sealants can be used to stopthedrainage.

The newborn during the 5 days of observation did not show any signs of pulmonary hypoplasia and this happens rarely among those with prolonged pre-viable PPROM according to various studies. However, long term sequelae for the newborn are significant including pulmonary hypertension, musculoskeletal abnormalities and central nervous system deficits. This therefore requires closer and prolonged follow up.

\section{Acknowledgements}

None.

\section{Conflict of interest}

Author declares that there is no conflict of interest.

\section{References}

1. Nederlandse Vereniging van Obstetrie en Gynaecologie [Rupture of the membranes before onset of labour].

2. Ernest JM. Neonatal consequences of preterm PPROM. Clin Obstet Gynecol. 1998;41(4):827-831.

3. Margato MF, Martins GL, Passini Júnior R, et al. Previable preterm rupture of membranes: gestational and neonatal outcomes. Arch Gynecol Obstet. 2012;285(6):1529-1534.

4. Van der Heyden JL, Van der Ham DP, Van Kuijk S, et al. Outcome of pregnancies with preterm prelabor rupture of membranes before 27 weeks' gestation: a retrospective cohort study. Eur J Obstet Gynecol Reprod Biol. 2013;170(1):125-130.

5. Hamar BD, Wolff EF, Kodaman PH, et al. Premature rupture of membranes, placenta increta, and hysterectomy in a pregnancy following endometrial ablation. J Perinatol. 2006;26(2):135-137.

6. Farooqi A, Holmgren PA, Engberg S, et al. Survival and 2-year outcome with expectant management of second-trimester rupture of membranes. Obstet Gynecol. 1998;92:895-901.
7. Botet F, Cararach V, Sentis J. Premature rupture of membranes in early pregnancy. Neonatal prognosis. J Perinat Med. 1994;22(1):45-52.

8. Vergani P, Ghidini A, Locatelli A, et al. Risk factors for pulmonary hypoplasia in second-trimester premature rupture of membranes. Am J Obstet Gynecol. 1994;170(5 Pt 1):1359-1364.

9. Morales WJ. The effect of chorioamnionitis on the developmental outcome of preterm infants at one year. Obstet Gynecol. 1987;70(2):183-186.

10. Alexander JM, Mercer BM, Miodovnik M, et al. The impact of digital cervical examination on expectantly managed preterm rupture of membranes. Am J Obstet Gynecol. 2000;183(4):1003-1007.

11. Gonen R, Hannah ME, Milligan JE. Does prolonged preterm premature rupture of the membranes predispose to abruptio placentae? Obstet Gynecol. 1989;74(3 Pt 1):347-350.

12. Mercer BM, Arheart KL. Antimicrobial therapy in expectant management of preterm premature rupture of the membranes. Lancet. 1995;346(8985):1271-1279.

13. Lemons JA, Bauer CR, Oh W, et al. Very low birth weight outcomes of the National Institute of Child Health and Human Development Neonatal Research Network, January 1995 through December 1996. NICHD Neonatal Research Network. Pediatrics. 2001;107(1):E1.

14. Spinillo A, Capuzzo E, Stronati M, et al. Effect of preterm premature rupture of membranes on neurodevelopmental outcome: follow up at two years of age. Br J Obstet Gynaecol. 1995;102(11):882-887.

15. Locatelli A, Ghidini A, Paterlini G, et al. Gestational age at preterm premature rupture of membranes: a risk factor for neonatal white matter damage. Am J Obstet Gynecol. 2005;193(3 Pt 2):947-951.

16. Moretti M, Sibai BM. Maternal and perinatal outcome of expectant management of premature rupture of the membranes in the midtrimester. Am J Obstet Gynecol. 1988;159(2):390-396.

17. Van Eyck J, van der Mooren K, Wladimiroff JW. Ductus arteriosus flow velocity modulation by fetal breathing movements as a measure of fetal lung development. Am J Obstet Gynecol. 1990;163(2):558-566.

18. Shumway J, Al-Malt A, Amon E, et al. Impact of oligohydramnios on maternal and perinatal outcomes of spontaneous premature rupture of the membranes at 18-28 weeks. J Matern Fetal Med. 1999;8(1):20-23.

19. ACOG Committee on Practice Bulletins. Acog Practice Bulletin: clinical management guidelines for obstetrician-gynecologists number 80 , april 2007. Obstet Gynecol. 2007;109(5):1233-1248. 